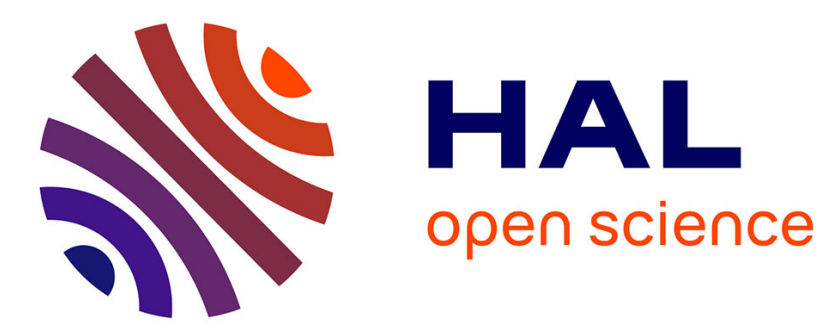

\title{
Vers une amélioration d'un modèle global pluie-débit au travers d'une approche comparative
}

\author{
Charles Perrin
}

\section{To cite this version:}

Charles Perrin. Vers une amélioration d'un modèle global pluie-débit au travers d'une approche comparative. La Houille Blanche - Revue internationale de l'eau, 2002, 6-7, pp.84-91. 10.1051/lhb/2002089 . hal-01098286

\section{HAL Id: hal-01098286 \\ https://hal.science/hal-01098286}

Submitted on 7 Jan 2015

HAL is a multi-disciplinary open access archive for the deposit and dissemination of scientific research documents, whether they are published or not. The documents may come from teaching and research institutions in France or abroad, or from public or private research centers.
L'archive ouverte pluridisciplinaire HAL, est destinée au dépôt et à la diffusion de documents scientifiques de niveau recherche, publiés ou non, émanant des établissements d'enseignement et de recherche français ou étrangers, des laboratoires publics ou privés. 


\title{
Vers une amélioration d'un modèle global pluie-débit au travers d'une approche comparative*
}

\author{
Towards an improved version of a lumped rainfall-runoff model \\ through a comparative approach
}

\author{
Charles Perrin
}

Cemagref

The study examined and evaluated 38 structures of lumped rainfall-runoff models on 429 catchments located in France. the United-States, Australia, the Ivory Coast and Brazil. All structures were evaluated at a daily time-step in the same testing framework and fed with the same amount of data. Their parameters were calibrated using a local optimisation search procedure that proved able to locate satisfactory sets of optimum parameters. Model performances in terms of quality of observed streamflow simulation were assessed with several quantitative criteria, including new formulations.

Tests results indicate that a low number of free parameters ( 3 to 5$)$ is sufficient to achieve a satisfactory modelling of the catchment hydrological behaviour, the increasing number of degrees of freedom in the model structure limiting model robustness. Furthermore model structure plays a key role in the quality of simulations. Some mathematical tools used in different structures appear complementary and their association in a single model may improve streamflow simulations. The structure of the simple GR3J model was thus modified into a more satisfactory four-parameter structure, with improved low flow simulations. Such a model can be used to solve many issues of water resources management and engineering.

\section{CONTEXTE ET OBJECTIFS}

Les gestionnaires de la ressource en eau doivent faire face aujourd'hui à des situations de plus en plus complexes, où interviennent de multiples acteurs, aux intérêts et objectifs parfois opposés et où existe une forte variabilité de la disponibilité en eau allant de situations de pénurie à des inondations catastrophiques.

On comprend alors aisément la nécessité de mettre au point des outils de gestion et d'aide à la décision, qui permettent de mieux cerner le fonctionnement des hydrosystèmes naturels et donc de mieux prévenir, anticiper et gérer les problèmes. Dans cette démarche d'appréhension des hydrosystèmes, la connaissance des débits des cours d'eau est un instrument de gestion indispensable. De nombreuses recherches se sont donc attachées, depuis plus d'un siècle, à comprendre les processus de génération des débits et le fonctionnement hydrologique du bassin versant. Un moyen pour simuler les débits est de remonter à leur cause première, les pluies. Ainsi, de nombreux modèles permettant d'obtenir une

* Cette thèse a reçu le prix Henri Milon 2002. représentation simplifiée de ce lien entre pluie et débit ont été construits, l'un des tout premiers ayant été le modèle de Stanford [1]. Ces modèles peuvent répondre à de nombreux problèmes opérationnels dans le domaine de la gestion de la ressource en eau et de l'ingénierie.

Le travail de recherche [2] présenté ici s'est inscrit dans le cadre de cette démarche de modélisation de la transformation de la pluie en débit et de sa représentation à l'échelle du bassin versant. L'objectif principal est d'essayer de mieux comprendre, au travers d'une approche comparative, le fonctionnement et la qualité des modèles simples de simulation des débits et de tenter d'en améliorer le fonctionnement. La partie II de cet article présente la méthodologie utilisée pour l'évaluation comparative des modèles pluie-débit existants, dont les résultats sont donnés en partie III. Sur cette base, la partie IV présente le travail d'amélioration réalisé sur l'un de ces modèles. La partie $\mathrm{V}$ présente succinctement une analyse sur les méthodes d'évaluation des incertitudes sur les paramètres et sur les méthodes de régionalisation des paramètres pour l'application des modèles sur les sites non jaugés. Enfin, la partie VI présente quelques unes des applications possibles de ces modèles pluie-débit. 


\section{II a MÉTHODOLOGIE DE COMPARAISON}

Le travail de comparaison entrepris avait pour but d'évaluer les forces et les faiblesses des modèles existants, afin d'identifier les points essentiels de la construction d'un modèle qui permettent d'obtenir une bonne représentation du comportement hydrologique du bassin.

L'appréciation des qualités et des faiblesses des modèles est délicate dans le cadre de simples exercices de validation individuels. Des études comparatives ayant impliqué plusieurs modèles (voir par exemple $[3,4,5,6,7]$ ) ont pu mieux mettre en évidence ces aspects. Cependant, les exercices de comparaison réalisés jusqu'à présent, en ne considérant qu'un faible nombre de modèles et de bassins test, ne permettent pas de dégager de conclusions claires quant aux valeurs respectives des différentes structures existantes.

Notre étude comparative avait pour but de dépasser les limites des études conduites jusqu'à présent en testant un grand nombre de structures de modèles sur un large échantillon de bassins versants, afin d'explorer le rôle du nombre de paramètres optimisables et celui de la formulation du modèle sur la qualité des simulations de débit. Cette démarche s'est appuyée sur un cadre de comparaison rigoureux dont nous détaillons ici les principaux aspects.

\section{II.1 Modèles}

Il existe aujourd'hui un grand nombre de modèles mathématiques de simulation de la transformation de la pluie en débit à l'échelle du bassin versant, parmi lesquels on trouve les modèles conceptuels ou empiriques globaux qui représentent le lien entre la pluie et le débit par des agencements variés de réservoirs et de fonctions mathématiques simples. Ces modèles correspondent à des idées variées de modélisateurs sur la façon de représenter le comportement du bassin versant.

Ici, nous avons retenu 38 structures dérivées de modèles existants (parmi lesquels on retrouve des modèles couramment utilisés tels que TOPMODEL [8], IHACRES [9], HBV [10], NAM [11], GR3J [12], etc.) et comprenant au maximum neuf paramètres libres pour les tester au pas de temps journalier. Ce regroupement de modèles programmés de façon semblable, très large sans cependant être exhaustif, offre la possibilité de réaliser des études systématiques sur le rôle réel de l'architecture mathématique du modèle et de son niveau de complexité (entendu comme le nombre de paramètres) sur ses performances.

\section{II.2 Bassins versants}

Ces structures ont été évaluées sur 429 bassins versants situés en France, aux Etats-Unis, en Australie, en Côted'Ivoire et au Brésil, pour lesquels nous disposions de chroniques journalières de pluie, d'évapotranspiration potentielle (ETP) et de débit. Une telle base de données, utilisée pour la première fois à cette échelle dans un exercice de comparaison, présente l'avantage de regrouper une grande diversité de caractéristiques climatiques, allant de conditions semi-arides à tropicales humides, et de types de bassin, avec un large éventail de superficies $\left(0,1\right.$ à $\left.50600 \mathrm{~km}^{2}\right)$, de couverts végétaux et de caractéristiques géologiques, pédologiques ou topographiques. Cette variété nous offrait la possibilité de tester la capacité des modèles à s'adapter à des conditions très différentes.

\section{II.3 Cadre comparatif}

Toutes les structures ont été placées dans un cadre homogène de comparaison fondé sur des tests de calages suivis de contrôles sur périodes indépendantes [13]. Au total, 1284 bassins-périodes, d'une longueur moyenne d'environ cinq années, ont été utilisées, ce qui a conduit à 3204 tests en contrôle sur l'échantillon de bassins. Les modèles ont reçu en entrée les mêmes données de pluie et d'évapotranspiration potentielle. Leurs paramètres ont été calés à l'aide d'une même procédure locale d'optimisation, appelée méthode "pas-à-pas " $[14,15]$. Une évaluation de cette méthode, réalisée sur quatre modèles de complexité différente, a montré qu'elle était efficace pour localiser des jeux de paramètres permettant d'obtenir des résultats satisfaisants du modèle. Cependant, ces tests ont également indiqué que l'accroissement de la complexité du modèle multipliait la présence d'optima équivalents dans l'espace des paramètres (rejoignant la notion «d'équifinalité ») [16].

Une même fonction objectif a été utilisée pour le calage des modèles. Il s'agit du critère de Nash-Sutcliffe [17], forme adimensionnelle d'un critère des moindres carrés des erreurs, calculé ici sur les racines carrées des débits et défini par:

$$
F(\%)=100 \cdot\left(1-\frac{\sum_{i=1}^{n}\left(\sqrt{Q_{o b s, i}}-\sqrt{Q_{c a l, i}}\right)^{2}}{\sum_{i=1}^{n}\left(\sqrt{Q_{o b s, i}}-\sqrt{M}\right)^{2}}\right)
$$

où $Q_{o b s, i}$ et $Q_{c a l, i}$ sont respectivement les débits observé et calculé au pas de temps $i, n$ le nombre de pas de temps de la

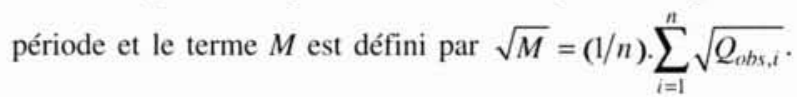
Dans ce critère relatif, $M$ peut être considéré comme un modèle de référence (ici une valeur constante à chaque pas de temps) auquel est comparé le modèle évalué. Ce critère peut prendre des valeurs entre $-\infty$ et $100 \%$, la valeur 0 représentant une qualité de simulation équivalente à celle du modèle de référence. La transformation préalable en racine carrée permet de ne pas donner trop de poids aux erreurs associées aux événements de crue lors du calage des paramètres du modèle.

L'évaluation des modèles a été faite en phase de contrôle uniquement, qui est plus proche des conditions d'utilisation opérationnelle des modèles que le calage. Pour avoir un point de vue objectif et complet sur les performances des modèles, nous avons utilisé un jeu de six critères quantitatifs complémentaires, tous écrits sous forme adimensionnelle et variant entre $-\infty$ et $100 \%$. A ce niveau, nous avons pu constater la difficulté de trouver des mesures relatives de quantification de l'erreur du modèle et nous avons mis en évidence les limites de pertinence du critère de NashSutcliffe, en particulier dans le cas de bassins où la variance initiale des débits est faible. Un critère relatif faisant intervenir un modèle de référence (correspondant à $M$ dans l'équation 1) fondé sur les entrées du modèle (c'est-à-dire sur la pluie et l'ETP, et non sur la moyenne des débits observés comme c'est le cas dans ce critère) et dérivant de travaux sur des modèles fonctionnant à grands pas de temps [18], a pu être proposé et est apparu plus général. 


\section{III $\square$ RÉSULTATS DES TESTS COMPARATIFS}

L'analyse des résultats a conduit à distinguer deux aspects majeurs de modélisation qui conditionnent la capacité des modèles conceptuels et empiriques globaux à simuler la transformation pluie-débit, à savoir la complexité et la formulation mathématique de leur structure [19].

Tout d'abord, afin d'avoir une base de référence pour évaluer les performances des modèles testés, nous avons utilisé un modèle plus rudimentaire ne comportant pas de procédure conceptuelle de suivi d'humidité du bassin. Le modèle utilisé ici est le modèle de Tsykin [20], qui est une équation non linéaire calculant le débit en fonction des pluies et de l'ETP antérieures. Une première conclusion de ces tests est que les modèles évalués apparaissent tous grossièrement équivalents lorsqu'ils sont comparés à ce modèle rudimentaire.

Les résultats de la comparaison ont indiqué que l'accroissement de la complexité du modèle (nombre de paramètres optimisés) améliore logiquement en phase de calage la capacité du modèle à simuler efficacement les débits. Cependant, en phase de contrôle, des structures simples avec seulement trois à cinq paramètres optimisés obtiennent des résultats aussi satisfaisants que des structures plus complexes, comme le montre la figure 1 . Le manque de robustesse des structures les plus complexes est essentiellement dû à leur sur-paramétrisation. Les gains de performance obtenus en calage par ajout de paramètres supplémentaires sont perdus en contrôle, démontrant ainsi le caractère factice du succès obtenu en calage.

La figure 1 indique également qu'à niveau de complexité identique, il peut exister d'importants écarts de performances entre des structures différentes. Cette analyse fait donc ressortir un autre aspect important de la construction des modèles : la formulation mathématique de la structure, c'està-dire le choix et l'agencement des réservoirs et des fonctions intervenant dans les modules de production et de routage du modèle, joue aussi un rôle essentiel sur les performances du modèle. A partir de l'approche comparative, nous avons montré que certaines structures de modèle, utilisant des fonctions différentes, sont complémentaires (en terme de performance) dans leur capacité à simuler les débits. Par ailleurs, pour visualiser les limites des gains de performance possibles, nous avons construit un modèle «idéal " multiforme, revenant à prendre sur chaque bassin la meilleure de toutes les structures testées. Ce modèle obtient des performances plus satisfaisantes que les meilleures des 38 structures testées, comme l'indique la figure 2 où sont représentées les distributions des résultats obtenus en contrôle pour les différents modèles. Ces éléments suggèrent que des progrès peuvent être faits dans la formulation des structures des modèles, conduisant à des structures plus performantes. Notons toutefois que, même pour ce modèle « idéal ", le critère d'efficacité passe une fois sur deux en dessous de $80 \%$, un seuil souvent considéré comme un objectif à dépasser pour qu'un modèle soit réellement efficace dans la pratique.

Les résultats montrent par ailleurs que les modèles globaux utilisés obtiennent sur les grands bassins des résultats aussi bons que sur des petits bassins. Leur nature globale n'est donc pas incompatible avec une application sur de grands bassins.

En regardant les performances des modèles suivant le type de climat considéré, on constate que des modèles initialement développés pour un type de climat particulier peuvent également obtenir de bons résultats dans des contextes climatiques très différents. Cette capacité d'adaptation des modèles, en partie liée à la souplesse donnée par les paramètres, laisse penser que les modèles restent souvent applicables en dehors des limites restreintes de conditions pour lesquelles leurs concepteurs les avaient initialement développés. Enfin, nous avons pu constater que pour presque chacun des modèles étudiés, on arrivait toujours à trouver au moins un bassin pour lequel le modèle surclassait tous les autres.

\section{IV — DÉMARCHE D'AMÉLIORATION DE MODÈLES}

La démarche d'amélioration de modèle s'est fondée sur les résultats des tests comparatifs exposés précédemment. Dans cette étude, le modèle GR3J [12] dont la structure
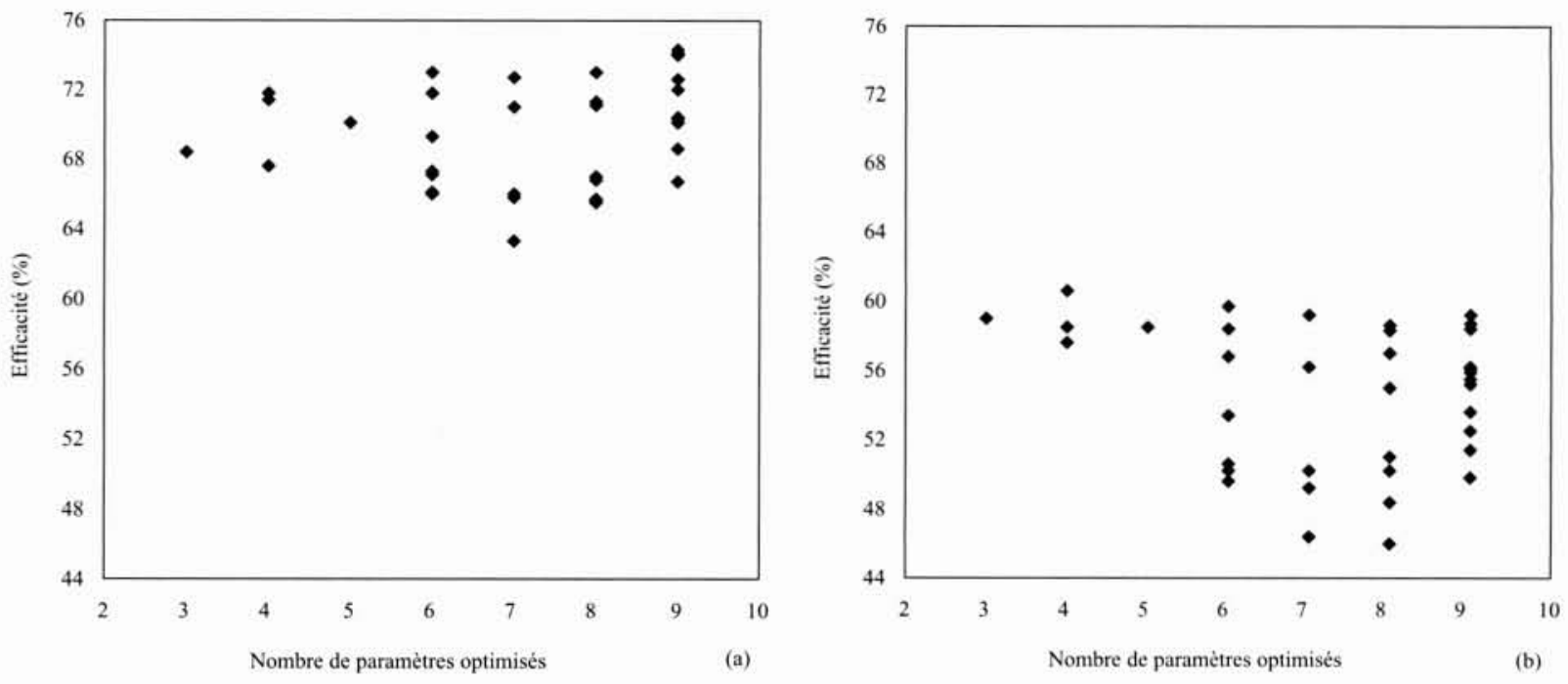

Figure 1 : Efficacités moyennes des modèles, sur l'échantillon des 429 bassins, en fonction du nombre de paramètres optimisés (a) au calage et (b) au contrôle. 


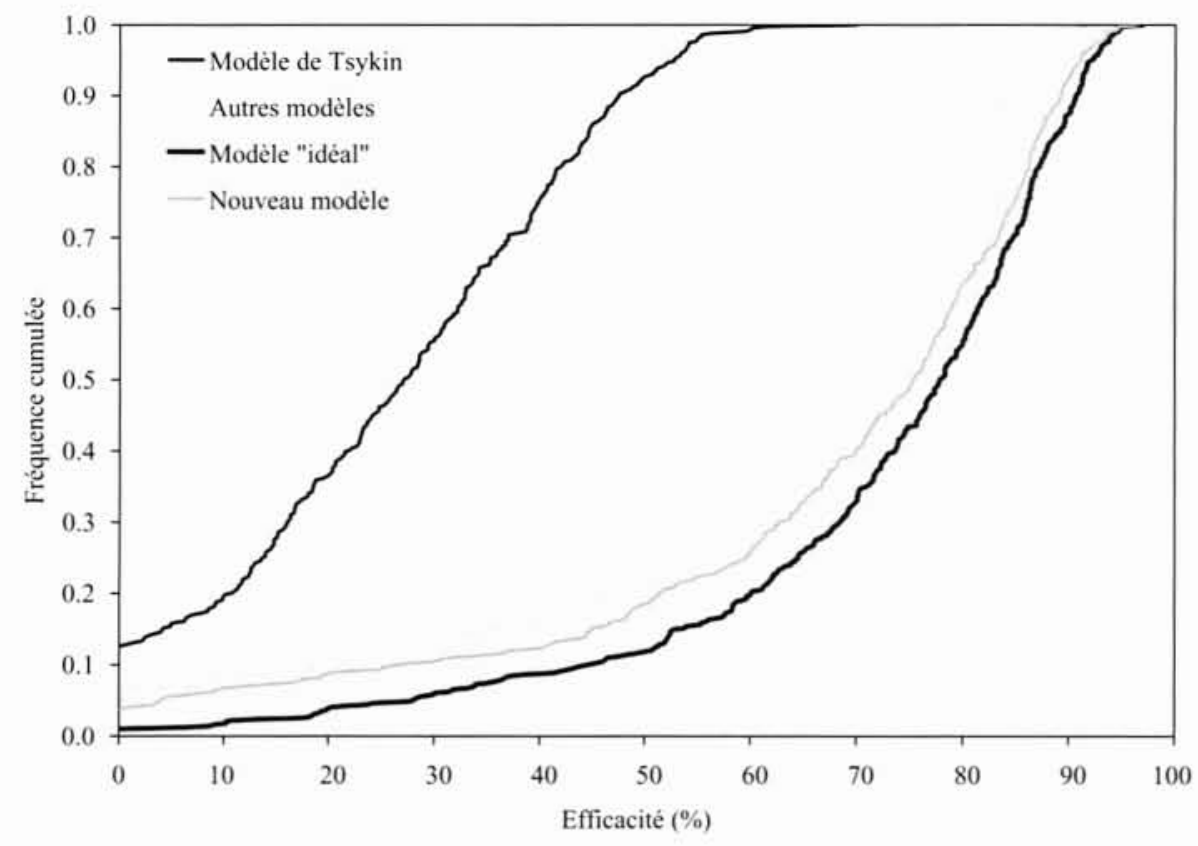

Figure 2 : Distribution des performances du modèle de Tsykin, du modèle « idéal ", des 38 structures de modèles testées et de la nouvelle version du modèle GR4J, sur l'échantillon des 429 bassins en contrôle.

dépend seulement de trois paramètres, est apparu parmi les plus fiables et les plus robustes, qualités provenant notamment de sa parcimonie et de l'approche empirique adoptée dans son développement, qui a permis de choisir les différents éléments de sa structure avec le seul souci d'efficacité. L'analyse des complémentarités avec d'autres structures de modèle a mis en évidence une voie possible de modification. En exploitant des fonctionnalités présentes dans d'autres modèles, nous avons pu rajouter un degré de complexité supplémentaire et proposer une structure modifiée du modèle. Celle-ci intègre en plus une percolation issue du réservoir de production (voir figure 3 et les détails mathématiques en Annexe), qui s'est révélée efficace. Cette version à quatre paramètres permet d'améliorer assez sensiblement les performances sur les différents critères de qualité par rapport aux versions antérieures du modèle : elle réalise un progrès substantiel sur la simulation des étiages et une légère amélioration sur la représentation des crues [21]. Le gain acquis en efficacité est illustré par la figure 2, avec une distribution des résultats pour cette nouvelle version plus proche de celle du modèle «idéal ». Les améliorations ainsi obtenues permettent d'avoir une confiance accrue dans le modèle et garantissent de ce fait une meilleure fiabilité aux applications hydrologiques qui l'utilisent.

\section{V घ INCERTITUDES SUR LES PARAMÈTRES ET RÉGIONALISATION}

Ces travaux ont également apporté des contributions méthodologiques à l'évaluation des incertitudes sur les paramètres des modèles pluie-débit et à la régionalisation de leurs paramètres.

Nous avons pu montrer qu'une méthode d'évaluation des incertitudes classiquement utilisée en hydrologie (méthode par approximation linéaire [22]) donnait des valeurs d'écarts-type sur les paramètres faibles en comparaison d'une méthode proposée dans le cadre de ce travail, reposant sur une procédure de calages multiples du modèle sur un
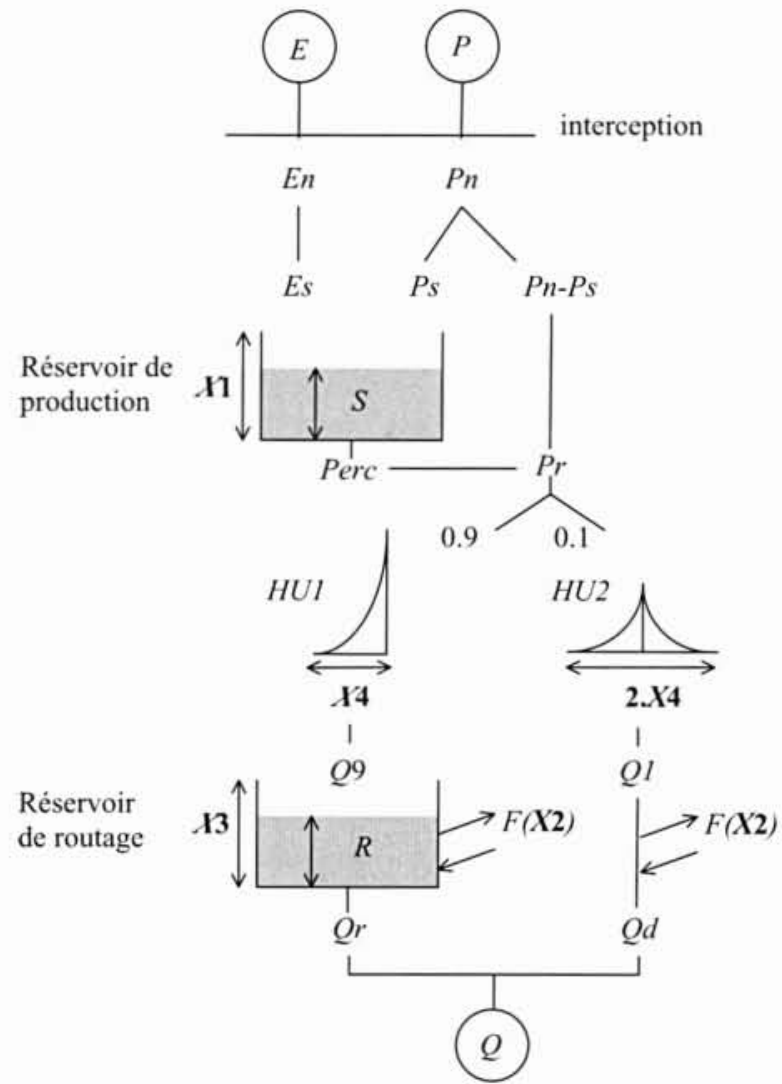

Figure 3 : Schéma de la structure de la nouvelle version du modèle GR4J.

grand nombre de périodes différentes. Ces résultats tendent à montrer que les incertitudes sur les paramètres des modèles pluie-débit sont généralement sous-estimées.

Par ailleurs, l'application des modèles pluie-débit sur des bassins versants non jaugés requiert l'établissement de liens entre valeurs des paramètres et descripteurs physiques et/ou 
climatiques du bassin par des études de régionalisation. Partant de l'analyse de la méthodologie généralement adoptée dans ce type d'études (voir par exemple [23, 24, 25]), nous avons pu mettre en évidence que ces études privilégiaient la qualité du lien entre valeurs des paramètres du modèle et descripteurs du bassin par rapport à la performance des modèles. Nous avons alors proposé une méthodologie alternative, qui privilégie la qualité de restitution des débits et qui permet donc d'obtenir des résultats du modèle sensiblement meilleurs dans un contexte d'application sur des bassins versants non jaugés. A l'issue d'une analyse n'introduisant que quelques descripteurs climatiques et la superficie des bassins versants, nous avons obtenu les résultats suivants pour le modèle GR4J sur l'échantillon des 429 bassins :

- Les valeurs médianes des quatre paramètres sont approximativement :

$$
\begin{aligned}
& (X 1)_{\text {med }}=400(\mathrm{~mm}) \\
& (X 2)_{\text {med }}=-0.6(\mathrm{~mm}) \\
& (X 3)_{\text {med }}=0.4\left(P_{m x}-P_{m n}\right)^{1.1}(\mathrm{~mm}) \\
& (X 4)_{\text {med }}=0.5+1.3 \frac{S^{0.16}}{P_{j y}^{0.64}}(\mathrm{j})
\end{aligned}
$$

où $P_{m x}$ et $P_{m n}$ sont les pluies mensuelles moyennes $(\mathrm{mm})$ respectivement du mois le plus arrosé et du mois le moins arrosé, $P_{j y}$ est la pluie journalière moyenne $(\mathrm{mm})$ et $S$ est la superficie du bassin versant $\left(\mathrm{km}^{2}\right)$.

- $\ln (X 1), \sinh (X 2), \ln (X 3)$ et $\ln (X 4-0.5)$ ont des distributions approximativement normales avec des écarts-types de 0.63 , $1.3,0.74$ et 0.55 respectivement.

\section{MODÉLISATION PLUIE-DÉBIT ET APPLICATIONS OPÉRATIONNELLES}

Ce travail de recherche s'inscrit dans une démarche de mise au point de modèles à la fois fiables et simples, en vue d'une utilisation pour répondre à des questions pratiques de gestion de la ressource en eau et d'ingénierie. Parmi les divers types de modèles existants, les modèles conceptuels ou empiriques globaux à peu de paramètres comme ceux étudiés ici, par leur robustesse et leur facilité d'utilisation, apparaissent les plus aptes à répondre aux questions telles que :

- la prévision de crue à court terme, avec utilisation conjointe d'une méthode de mise à jour du modèle. Prévoir les crues en remontant aux pluies permet notamment d'augmenter les délais de prévision par rapport aux approches classiques de propagation [26] ;

- la prévision d'étiages à long terme, avec utilisation conjointe d'un générateur stochastique de pluie [27] ;

- la prédétermination des crues, avec également un générateur stochastique de pluies $[28,29]$;

- la détection d'impacts sur les débits dus à des aménagements ou des changements d'occupation des sols [30] ;

- le dimensionnement des réservoirs d'écrêtement de crue et/ou de soutien d'étiage et leur gestion [31].
Nous pensons par ailleurs que ces modèles simples, par la qualité des simulations des volumes d'eau qu'ils peuvent fournir, devraient pouvoir contribuer efficacement à d'autres domaines connexes tels que la modélisation de la qualité des eaux.

\section{VII $\square$ CONCLUSION}

Ce travail a montré que, malgré les efforts réalisés depuis plusieurs décennies en modélisation pluie-débit, des améliorations étaient encore possibles. Il laisse entrevoir que d'autres améliorations substantielles peuvent encore être réalisées dans ce domaine de la modélisation pluie-débit à l'échelle du bassin versant, notamment par l'exploitation de démarches comparatives.

A côté de ces perspectives de recherches, ce travail nous permet d'engager avec plus de confiance le développement d'outils hydrologiques s'appuyant sur le modèle GR4J et destinés à être utilisés dans le domaine opérationnel. Il existe en effet une forte demande dans ce sens en France et à l'étranger de la part des ingénieurs, des concepteurs d'ouvrages, des gestionnaires de la ressource et des décideurs pour des applications telles que la prévision des crues, la prévision d'étiage à long terme, le dimensionnement et la gestion de réservoirs, la prédétermination des crues, la détection de changements de comportement hydrologique d'un bassin, questions auxquelles la modélisation pluie-débit est aujourd'hui en mesure d'apporter assez souvent une réponse utile. Pour toutes ces applications, une approche fondée sur une méthodologie d'évaluation comparative telle que celle proposée dans ce travail paraît indispensable.

\section{REMERCIEMENTS}

Je tiens à remercier Jean-Michel Grésillon, directeur de l'Ecole Nationale Supérieure d'Hydraulique et de Mécanique de Grenoble (ENSHMG) (maintenant au Cemagref) pour avoir dirigé cette thèse; Claude Michel, hydrologue de l'unité de recherche Qualité et fonctionnement hydrologique des systèmes aquatiques (QHAN) au Cemagref d'Antony, pour avoir encadré ces travaux : les membres de mon comité de thèse, pour leurs conseils; les membres de l'unité QHAN, en particulier Vazken Andréassian, pour leur soutien. Ce travail de thèse s'inscrit dans l'école doctorale Terre, Univers, Environnement de l'Institut National Polytechnique de Grenoble (INPG), dans le cadre d'une Formation Continue Par la Recherche (FCPR) de l'Ecole Nationale du Génie de l'Eau et de l'Environnement de Strasbourg (ENGEES). Je remercie l'ENGEES et le Cemagref pour leur soutien pour ces travaux.

Je remercie également les personnes ayant fourni des données pour mener à bien cette étude: Dr Francis H.S. Chiew (University of Melbourne, Australie), Dr Eric Servat (IRD, Montpellier), Jane L. Thurman (US Department of Agriculture, Beltsville, Etats-Unis), Dr Nilo Oliveira Nascimento (University of Minas Gerais, Belo Horizonte, Brésil). Des données de la base MOPEX (Site Web : www.nws.noaa.gov/ oh/mopex) et des bases de données hydrométriques HYDRO et pluviométriques PLUVIO en France ont été utilisées dans ce travail. 


\section{BIBLIOGRAPHIE}

[1] CRAwFORD N.H., LinsLey R.K. (1963), - « A conceptual model of the hydrologic cycle $n$. IAHS Publication $n^{\circ} 63,573-$ 587.

[2] Perrin C. (2000), - «Vers une amélioration d'un modèle global pluie-débit au travers d'une approche comparative ", Thèse de Doctorat, INPG (Grenoble) / Cemagref (Antony), 530 p.

[3] WMO (1975). - « Intercomparison of conceptual models used in operational hydrological forecasting ", Operational Hydrology Report n* 7, WMO n" 429, World Meteorological Organization, Geneva, Switzerland.

[4] Franchini M., PaCCiani M. (1991), - «Comparative analysis of several conceptual rainfall-runoff models ", Journal of Hydrology, 122, 161-219.

[5] Chiew F.H.S., Stewardson M.J., McMahon T.A. (1993), "Comparison of six rainfall-runoff modelling approaches", Journal of Hydrology, 147, 1-36.

[6] Gan T.Y., Dlamini E.M., Biftu G.F. (1997). - « Effects of model complexity and structure, data quality and objective function on hydrologic modeling ". Journal of Hydrology, 192, 81-103.

[7] Perrin C., Littlewood 1.G. (2000). - «A comparative assessment of two rainfall-runoff modelling approaches: GR4J and IHACRES ", Proceedings of the Liblice Conference (2224 September 1998), V. Elias and I.G. Littlewood (Ed.), IHP$\mathrm{V}$. Technical Documents in Hydrology n* 37 , UNESCO, Paris, 191-201.

[8] BeVEN K.J.. KIRKBY M.J. (1979), - $\propto$ A physically based, variable contributing area model of basin hydrology n, Hydrological Sciences Bulletin, 24(1), 43-69.

[9] JaKeman A.J., Littl.eWood I.G., WhiteheAd P.G. (1990). « Computation of the instantaneous unit hydrograph and identifiable component flows with application to two small upland catchments ", Journal of Hydrology, 117, 275-300.

[10] Bergstróm S., Forsman A. (1973). - « Development of a conceptual deterministic rainfall-runoff model ", Nordic Hydrology, 4, 147-170.

[11] Nielsen S.A., HANSEN E. (1973). - « Numerical simulation of the rainfall-runoff process on a daily basis ", Nordic Hydro$\log y, 4,171-190$.

[12] Edjatno, Nascimento N.O., Yang X., Makhlouf Z., Michel. C. (1999), - « GR3J : a daily watershed model with three free parameters ", Hydrological Sciences Journal, 44(2), 263-277.

[13] KLEMES V. (1986). - $\propto$ Operational testing of hydrological simulation models ", Hydrological Sciences Journal, 31(1), 13 24.

[14] MiCHEL C. (1989). - « Hydrologie appliquée aux petits bassins versants ruraux ", Cemagref, Antony, 528 p.

[15] Nascimento N.O. (1995). - «Appréciation à l'aide d'un modèle empirique des effets d'action anthropiques sur la relation pluie-débit à l'échelle du bassin versant ». Thèse de Doctorat, CERGRENE/ENPC, Paris, $550 \mathrm{p}$.

[16] Beven K.J. (1993). - « Prophecy, reality and uncertainty in distributed hydrological modelling ", Advances in Water Resources, 16, 41-51.
[17] NASH J.E., SUTCL.IFFE J.V. (1970). - « River flow forecasting through conceptual models. Part I : A discussion of principles ", Journal of Hydrology, 27(3), 282-290.

[18] Mouel.hi S. (2000). - « Modélisation pluie-débit aux pas de temps mensuel, annuel et interannuel ", Rapport interne d'avancement, Thèse en cours, Cemagref, 58 p.

[19] Perrin C., Michel C., Andréassian V. (2001). - « Does a large number of parameters enhance model performance ? Comparative assessment of common catchment model structures on 429 catchments », Journal of Hydrology, 242(3-4), 275 301.

[20] TsYkin E.N. (1985). - $-\propto$ Multiple nonlinear statistical models for runoff simulation and prediction ", Journal of Hydrology, 77. 209-226.

[21] Perrin C., Michel C., Andréassian V. (2002). - « Improvement of a parsimonious model for streamflow simulation ", Journal of Hydrology, Soumis pour publication.

[22] Mein R.G., Brown B.M. (1978), - « Sensitivity of optimized parameters in watershed models ", Water Resources Research, 14(2), 299-303.

[23] Servat E., Dezetter A. (1993), - « Rainfall-runoff modelling and water resources assessment in northwestern Ivory Coast. Tentative extension to ungauged catchments ", Journal of Hydrology, 148, 231-248.

[24] Post D.A., Jakeman A.J. (1999). - « Predicting the daily streamflow of ungauged catchments in S.E. Australia by regionalising the parameters of a lumped conceptual model ", Ecological Modelling, 123, 91-104.

[25] YU P.S., YANG T.C. (2000). - $-\propto$ Using synthetic flow duration curves for rainfall-runoff model calibration at ungauged sites ", Hydrological Processes, 14(2), 117-133.

[26] YANG X., Michel C. (2000). — «Flood forecasting with a watershed model: a new method of parameter updating ", Hydrological Sciences Journal, 45(4), 537-546.

[27] Perrin C., Michel. C., Andréassian V. (2001). - « Longterm low flow forecasting for French rivers by continuous rainfall-runoff modelling ", Meeting of the British Hydrological Society on Continuous River Flow Simulation, Wallingford, UK. 5th July 2001, BHS Occasional Paper, n 13, I.G. Littlewood (Ed.), 21-29.

[28] LAMB R. (1999). - «Calibration of a conceptual rainfallrunoff model for flood frequency estimation by continuous simulation ”, Water Resources Research, 35(10), 3103-3114.

[29] Perrin C., Michel. C. (2002). - « Robustness of two flood estimation methods with data availability $n$, International Conference on Flood Estimation, Berne, Switzerland, in press.

[30] ANDRÉASSIAN V. (2002), - " Impact de l'évolution du couvert forestier sur le comportement hydrologique des bassins versants ". Thèse de Doctorat, Université Pierre et Marie Curie Paris VI, Cemagref (Antony), 276 p.

[31] Yang X., Parent E., Michel. C., Roche P.A. (1995). "Comparison of real-time reservoir-operation techniques", Journal of Water Resources Planning and Management, 121(5), 345-351. 


\section{ANNEXE : DESCRIPTION DU MODÈLE PLUIE-DÉBIT GR4J}

Le modèle GR 4 J (modèle du Génie R Rural à $\underline{4}$ paramètres Journalier) est un modèle pluie-débit global à quatre paramètres. La version développée dans le cadre de ces travaux est décrite dans ce qui suit. Une version Fortran est disponible à l'adresse suivante :

http ://www.antony.cemagref.fr/webqhan/projets\%20themes/ Hydrologie/Code\% 20fortran.htm

Dans la suite, on désignera par $P(\mathrm{~mm})$ la hauteur de pluie et par $E(\mathrm{~mm})$ l'évapotranspiration potentielle (ETP). $P$ est une estimation de la pluie de bassin et $E$ peut-être issue d'une courbe d'ETP moyenne interannuelle. Les équations suivantes correspondent aux équations intégrées sur un pas de temps.

La première opération est l'interruption de $P$ par $E$ pour déterminer une pluie nette $P n$ et une évapotranspiration nette En calculée par :

$$
\begin{array}{ll}
\text { Si } P \geq E \text {, alors } P n=P-E & \text { et } E n=0 \\
\text { Si } P<E \text {, alors } P n=0 & \text { et } E n=E-P
\end{array}
$$

Dans le cas où $P n$ est différente de zéro, une partie $P s$ de $P n$ alimente le réservoir de production et est calculée par :

$$
P s=\frac{X 1 \cdot\left(1-\left(\frac{S}{X 1}\right)^{2}\right) \cdot \tanh \left(\frac{P n}{X 1}\right)}{1+\frac{S}{X 1} \cdot \tanh \left(\frac{P n}{X 1}\right)}
$$

où $X 1(\mathrm{~mm})$ est la capacité maximum du réservoir de production.

Dans le cas contraire, lorsque En est différent de zéro, une quantité d'évaporation Es est retirée du réservoir de production. Elle est donnée par:

$$
E s=\frac{S \cdot\left(2-\frac{S}{X 1}\right) \cdot \tanh \left(\frac{E n}{X 1}\right)}{1+\left(1-\frac{S}{X 1}\right) \cdot \tanh \left(\frac{E n}{X 1}\right)}
$$

Le contenu du réservoir est mis à jour par :

$$
S=S-E s+P s
$$

Une percolation Perc issue du réservoir de production est alors calculée par l'équation suivante :

$$
\text { Perc }=S .\left\{1-\left[1+\left(\frac{4}{9} \frac{S}{X 1}\right)^{4}\right]^{-\frac{1}{4}}\right\}
$$

Le contenu du réservoir est mis à jour par :

$$
S=S-\text { Perc }
$$

et la quantité d'eau $\operatorname{Pr}$ qui atteint finalement la partie routage du modèle est donnée par:

$$
\operatorname{Pr}=\operatorname{Perc}+(P n-P s)
$$

$\operatorname{Pr}$ est divisée en deux composantes d'écoulement, $90 \%$ étant routés par un hydrogramme unitaire $H U 1$ et un réservoir de routage et $10 \%$ par un unique hydrogramme unitaire $H U 2$. HU1 et $H U 2$ dépendent du même paramètre $X 4$, temps de base de $H U 1$ exprimé en jours.

Les ordonnées des hydrogrammes sont calculées à partir des courbes en S notées respectivement $\mathrm{SH} 1$ et $S H 2$. $S H 1$ est définie en fonction du temps par:

Pour $t \leq 0$,

$$
S H 1(t)=0
$$

Pour $0<t<X 4, \quad S H 1(t)=\left(\frac{t}{X 4}\right)^{\frac{5}{2}}$

Pour $t \geq X 4$,

$$
S H 1(t)=1
$$

SH2 est définie de façon similaire par:

Pour $t \leq 0$

$$
\operatorname{SH} 2(t)=0
$$

Pour $0<t \leq X 4, \quad S H 2(t)=\frac{1}{2}\left(\frac{t}{X 4}\right)^{\frac{5}{2}}$

Pour $X 4<t<2 . X 4, \quad S H 2(t)=1-\frac{1}{2}\left(2-\frac{t}{X 4}\right)^{\frac{5}{2}}$

Pour $t \geq 2 . X 4$

$$
\text { SH } 2(t)=1
$$

Les ordonnées de $H U 1$ et $H U 2$ sont alors calculées par:

$$
\begin{gathered}
U H 1(j)=S H 1(j)-S H 1(j-1) \\
U H 2(j)=S H 2(j)-S H 2(j-1)
\end{gathered}
$$

où $j$ est un entier.

Un échange souterrain en eau est calculé par :

$$
F=X 2 .\left(\frac{R}{X 3}\right)^{7 / 2}
$$

où $R$ est le niveau dans le réservoir de routage, $X 3$ la capacité à un jour du réservoir et $X 2$ le coefficient d'échange en eau qui peut être positif dans le cas d'apports, négatif dans le cas de pertes vers des nappes profondes ou nul.

Le niveau dans le réservoir de routage est mis à jour en ajoutant la sortie $Q 9$ de l'hydrogramme $H U 1$ et $F$ :

$$
R=\max (0 ; R+Q 9+F)
$$

Il se vidange ensuite en une sortie $Q r$ donnée par:

$$
Q r=R \cdot\left\{1-\left[1+\left(\frac{R}{X 3}\right)^{4}\right]^{-\frac{1}{4}}\right\}
$$

Le niveau dans le réservoir devient:

$$
R=R-Q r
$$


La sortie $Q 1$ de l'hydrogramme $H U 2$ est soumise aux mêmes échanges pour donner la composante d'écoulement $Q d$ :

$$
Q d=\max (0 ; Q 1+F)
$$

Le débit total $Q$ est alors donné par :

$$
Q=Q r+Q d
$$

Le modèle a quatre paramètres optimisables : $X 1$ : capacité du réservoir de production $(\mathrm{mm})$ $X 2$ : coefficient d'échanges souterrains $(\mathrm{mm})$ $X 3$ : capacité à un jour du réservoir de routage $(\mathrm{mm})$ $X 4$ : temps de base de l'hydrogramme unitaire $H U 1$ (j) 